IMA Conference on Mathematics of Robotics

9 - 11 September 2015, St Anne's College, University of Oxford

\title{
Global Formulations of Lagrangian and Hamiltonian Dynamics on Embedded Manifolds
}

\author{
By Taeyoung Lee*, Melvin Leok ${ }^{\dagger}$, and N. Harris McClamroch ${ }^{\ddagger}$ \\ * Mechanical and Aerospace Engineering, George Washington University, Washington, DC 20052, USA, \\ ${ }^{\dagger}$ Mathematics, University of California, San Diego, La Jolla, CA 92093, USA \\ ${ }^{\ddagger}$ Aerospace Engineering, University of Michigan, Ann Arbor, MI 48109, USA.
}

\begin{abstract}
This paper provides global formulations of Lagrangian and Hamiltonian variational dynamics evolving on a manifold embedded in $\mathbb{R}^{n}$, which appears often in robotics and multi body dynamics. Euler-Lagrange equations and Hamilton's equations are developed in a coordinate-free fashion on a manifold, without relying on local parameterizations that may lead to singularities and cumbersome equations of motion. The proposed intrinsic formulations of Lagrangian and Hamiltonian dynamics are expressed compactly, and they are useful in analysis and computation of the global dynamics. These are illustrated by dynamic systems on the unit-spheres and the special orthogonal group.
\end{abstract}

\section{Introduction}

The configuration of various robotics can be represented by the elements of the one-sphere, the two-sphere, the special orthogonal group, or their products, which are manifolds embedded in $\mathbb{R}^{n}$. For example, the configuration of articulated robotic arms interconnected by spherical joints is represented by two-spheres (Murray 1993), and the configuration of unmanned aerial vehicles is defined by the special Euclidean group. In quantum mechanics, the pure state space of a twolevel quantum mechanical system is a two-sphere, referred to as the Bloch sphere (Bloch 1946).

In most of the existing literature on dynamical systems evolving on an embedded manifold, local coordinates are repeatedly used for dynamic modeling and control system design. For example, the dynamics of a spherical pendulum is often defined in terms of two angles that are typically considered to evolve on $\mathbb{R}^{2}$. However, such parameterizations of an embedded manifold suffer from the following two main issues. First, parameterizations represent the manifold only locally. This causes a singularity in representing the kinematics on the embedded manifold, which cannot be avoided unless one switches coordinate charts whenever the trajectory reaches the vicinity of the singularity.

The second issue is that the equations of motion of dynamical systems on an embedded manifold become exceedingly complicated when expressed using local coordinates. For example, the dynamics of a multiple spherical pendulum, written in terms of angles, involves complicated expressions of trigonometric functions. As such, it is impossible to develop the equations of motion for complex multibody systems in a concise manner in terms of local coordinates.

This paper provides global formulations of dynamics evolving on a manifold embedded in $\mathbb{R}^{n}$. In particular, we study dynamical systems that can be viewed as Lagrangian systems or as Hamiltonian systems that encompass a large class of mechanical systems that appear in robotics, structural dynamics, quantum mechanics or meteorology. Most importantly, dynamics are formulated directly on the manifold in a global fashion via variational principles.

This geometric formulation is said to be coordinate-free, as it does not require the use of 
IMA Conference on Mathematics of Robotics

9 - 11 September 2015, St Anne's College, University of Oxford

Global Formulations of Lagrangian and Hamiltonian Dynamics on Embedded Manifolds

local charts, coordinates or parameters that may lead to singularities or ambiguities in the representation. As such, it can be applied to arbitrarily large maneuvers on the manifold globally. Furthermore, this provides an efficient and elegant way to formulate, analyze, and compute the dynamics and their temporal evolutions. The corresponding mathematical model developed on the manifold is nicely structured and elegant. This representational efficiency has a substantial practical advantage compared with local coordinates for many complex dynamical systems; this fact has not been appreciated by the applied scientific and engineering communities.

In short, the main contribution of this paper is providing geometric formulations of the equations of motion for Lagrangian and Hamiltonian systems that evolve on an embedded manifold using variational methods. The proposed global formulations, that do not require local charts, have not been previously studied, even in the well-known literature on geometric mechanics, such as (Marsden 1992), (Holm 2009), (Marsden 1999). Preliminary results have been given for two-spheres in (Lee 2009), (Lee 2012), and for special Euclidean group in (Lee 2007). This paper provides both Euler-Lagrange equations and Hamilton's equations for an arbitrary embedded manifold, such as a subspace, the one-sphere, the two-sphere or the special orthogonal group.

Geometric mechanics, as presented in this paper, emphasizes the development of Lagrangian and Hamiltonian equations of motion in specific forms based on the geometric properties of the configuration manifold. This perspective differs from much of the existing literature on geometric mechanics, for example, such as in (Arnold 1989), (Bloch 2003), (Bullo 2005), (Marsden 1992), (Marsden 1990), where geometry is used in the analysis of solutions and flow properties.

\section{Lagrangian and Hamiltonian Dynamics on an Embedded Manifold}

\subsection{Variations on a manifold}

Consider a differentiable manifold $\mathrm{M}$ embedded in $\mathbb{R}^{n}$; we denote the tangent space at $x \in \mathrm{M}$ by $\mathrm{T}_{x} \mathrm{M}$ and we denote that tangent bundle of $\mathrm{M}$ by $\mathrm{TM}$. The cotangent space $\mathrm{T}_{x}^{*} \mathrm{M}$ is identified with $\mathrm{T}_{x} \mathrm{M}$ via the standard inner product on $\mathbb{R}^{n}$. The subsequent development describes variations of functions with values in the manifold $\mathrm{M}$. Let $x:\left[t_{0}, t_{f}\right] \rightarrow \mathrm{M}$ be a differentiable curve. The family of variations of $x$ is defined by a differentiable mapping $x^{\epsilon}:(-c, c) \times\left[t_{0}, t_{f}\right] \rightarrow \mathrm{M}$ for $c>0$; since $\mathrm{M}$ is an embedded manifold, each member of the family can be shown to have the power series form

$$
x^{\epsilon}(t)=x(t)+\epsilon \delta x(t)+\mathcal{O}\left(\epsilon^{2}\right), \quad t_{0} \leq t \leq t_{f},
$$

for some differentiable curve $\delta x:\left[t_{0}, t_{f}\right] \rightarrow \mathbb{R}^{n}$ that satisfies $\delta x(t) \in \mathrm{T}_{x(t)} \mathrm{M}, t_{0} \leq t \leq t_{f}$, and $\delta x\left(t_{0}\right)=\delta x\left(t_{f}\right)=0$. It is easy to see that the variations satisfy $x^{0}(t)=x(t), t_{0} \leq t \leq t_{f}$, and $x^{\epsilon}\left(t_{0}\right)=x\left(t_{0}\right)$ and $x^{\epsilon}\left(t_{f}\right)=x\left(t_{f}\right)$ for any $\epsilon \in(-c, c)$.

\subsection{Euler-Lagrange equations}

Consider a Lagrangian $L: \mathrm{TM} \rightarrow \mathbb{R}^{1}$. We derive the corresponding Euler-Lagrange equations of motion according to Hamilton's principle, namely that the infinitesimal variation of the action integral is zero along any motion as follow. Define the action integral along a motion that evolves on the manifold $\mathrm{M}$ as $\mathfrak{G}=\int_{t_{0}}^{t_{f}} L(x, \dot{x}) d t$.

The infinitesimal variation of the action integral can be written as

$$
\delta \mathfrak{G}=\int_{t_{0}}^{t_{f}}\left\{\frac{\partial L(x, \dot{x})}{\partial \dot{x}} \cdot \delta \dot{x}+\frac{\partial L(x, \dot{x})}{\partial x} \cdot \delta x\right\} d t .
$$

Integrating the first term on the right by parts, and using the fact that $\delta x$ vanishes at $t=t_{0}$ and 


\section{IMA Conference on Mathematics of Robotics}

\section{9-11 September 2015, St Anne's College, University of Oxford}

\section{LAGRANGIAN AND HAMILTONIAN DYNAMICS ON AN EMBEDDED MANIFOLD}

$t_{f}$, the infinitesimal variation of the action integral is given by

$$
\delta \mathfrak{G}=\int_{t_{0}}^{t_{f}}\left\{-\frac{d}{d t}\left(\frac{\partial L(x, \dot{x})}{\partial \dot{x}}\right)+\frac{\partial L(x, \dot{x})}{\partial x}\right\} \cdot \delta x d t .
$$

From Hamilton's principle, $\delta \mathfrak{G}=0$ for all infinitesimal variations $\delta x:\left[t_{0}, t_{f}\right] \rightarrow \mathbb{R}^{n}$, that satisfy $\delta x(t) \in \mathrm{T}_{x(t)} \mathrm{M}$ and vanish at $t_{0}$, and $t_{f}$. The fundamental lemma of the calculus of variations on a configuration manifold $\mathrm{M}$ implies that

$$
\left\{\frac{d}{d t}\left(\frac{\partial L(x, \dot{x})}{\partial \dot{x}}\right)-\frac{\partial L(x, \dot{x})}{\partial x}\right\} \cdot \delta x=0,
$$

for all $\delta x \in \mathrm{T}_{x} \mathrm{M}$. This is an abstract condition, essentially in the form given in (Arnold 1989), that characterizes the Lagrangian flow that evolves on the tangent bundle of the configuration manifold.

It is more convenient to express this Euler-Lagrange condition in the form of differential equations, defined on the embedding space $\mathbb{R}^{n}$, that describe this evolution on the tangent bundle $\mathrm{TM}$. To this end, we introduce the orthogonal projection matrix for each $x \in \mathrm{M}$ as $P(x): \mathbb{R}^{n} \rightarrow$ $\mathrm{T}_{x} \mathrm{M}$ which satisfies the orthogonality condition: for each $y \in \mathbb{R}^{n}$

$$
(y-P(x) y) \cdot z=0 \text {, for all } z \in \mathrm{T}_{x} \mathrm{M} .
$$

We assume the orthogonal projection operator $P$ is a differentiable matrix valued function. One may show that the projection operator $P(x)$ is symmetric, i.e., $P(x)=P(x)^{T} P(x)$.

Thus the Euler-Lagrange condition (2.1) holds for all infinitesimal variations at $x \in \mathrm{M}$ given by $\delta x=P(x) y$ for any $y \in \mathbb{R}^{n}$. This implies that the Euler-Lagrange condition can be expressed in terms of the orthogonal projection operator as follows.

Proposition 1. Consider a differentiable manifold $\mathrm{M}$ embedded in $\mathbb{R}^{n}$. The Euler-Lagrange equation for a Lagrangian $L: \mathrm{TM} \rightarrow \mathbb{R}^{1}$ can be written as

$$
P^{T}(x)\left\{\frac{d}{d t}\left(\frac{\partial L(x, \dot{x})}{\partial \dot{x}}\right)-\frac{\partial L(x, \dot{x})}{\partial x}\right\}=0,
$$

where the projection operator is defined by (2.2).

This is a more explicit characterization of the Lagrangian flow that evolves on the tangent bundle of the configuration manifold, that is TM. It is interesting to note that the expression in the braces corresponds to the conventional Euler-Lagrange equation for a mechanical system evolving on $\mathbb{R}^{n}$; the Euler-Lagrange equation on an embedded manifold is obtained via projection onto the tangent space.

\subsection{Legendre transformation and the Hamiltonian}

For the given Lagrangian, the Legendre transformation $\mathbb{F} L: \mathrm{TM} \rightarrow \mathrm{T}^{*} \mathrm{M}$ is defined as

$$
\mathbb{F} L(x, \dot{x}) \cdot v=\left.\frac{d}{d s}\right|_{s=0} L(x, \dot{x}+s v)=\mu \cdot v,
$$

where $v \in \mathrm{T}_{x} \mathrm{M}$. This yields the momentum vector $\mu \in \mathrm{T}_{x}^{*} \mathrm{M}$ given by

$$
\mu=P^{T}(x) \frac{\partial L(x, \dot{x})}{\partial \dot{x}} .
$$

We assume that the Lagrangian is regular or nondegenerate such that the Legendre transformation is locally invertible, which induces an equivalent Hamiltonian system on $\mathrm{T}^{*} \mathrm{M}$. Define the Hamiltonian function $H: \mathrm{T}^{*} \mathrm{M} \rightarrow \mathbb{R}^{1}$ as

$$
H(x, \mu)=\mu \cdot \dot{x}-L(x, \dot{x}) .
$$




\section{IMA Conference on Mathematics of Robotics}

\section{9-11 September 2015, St Anne's College, University of Oxford}

Global Formulations of Lagrangian and Hamiltonian Dynamics on Embedded Manifolds

One may derive Hamilton's equations by rewriting the Euler-Lagrange equation (2.3) in terms of $(x, \mu)$ via (2.4). However, following such procedure for an arbitrary manifold is quite challenging. As such, we derive Hamilton's equations directly from Hamilton's phase space variational principle instead.

\subsection{Hamilton's Equations}

Hamilton's equations on manifolds can be most directly obtained from a variational principle on $T^{*} \mathrm{M}$. Hamilton's phase space variational principle states that the infinitesimal variation of the action integral,

$$
\mathfrak{G}=\int_{t_{0}}^{t_{f}}\{\mu \cdot \dot{x}-H(x, \mu)\} d t
$$

subject to fixed endpoints for $x(t)$, along any motion is zero.

Integrating by parts, and using the fact that the variations vanish at the end points,

$$
\delta \mathfrak{G}=\int_{t_{0}}^{t_{f}}\left\{\left(-\dot{\mu}-\frac{\partial H(x, \mu)}{\partial x}\right) \cdot \delta x+\left(\dot{x}-\frac{\partial H(x, \mu)}{\partial \mu}\right) \cdot \delta \mu\right\} d t .
$$

Since the manifold $\mathrm{M}$ is embedded in $\mathbb{R}^{n}$, we have a constraint on the momentum $\mu$, which is given by $\left(I_{n \times n}-P(x)^{T}\right) \mu=0$. This follows from the fact that the projection $P(x)^{T}$ is idempotent, i.e., $P(x)^{T} P(x)^{T}=P(x)^{T}$. Therefore, by the definition (2.4) of $\mu, P(x)^{T} \mu=\mu$, or equivalently, $\left(I_{n \times n}-P(x)^{T}\right) \mu=0$. This induces a constrained variation,

$$
-\left(\frac{\partial P^{T}(x) \mu}{\partial x}\right) \delta x+\left(I_{n \times n}-P(x)^{T}\right) \delta \mu=0 .
$$

Then, the first term of the above equation can be viewed as a weighted linear combination of matrices acting on $\mu$. But, it is also linear in $\delta x$, and can be rewritten as a matrix acting on $\delta x$,

$$
\left(\frac{\partial P^{T}(x) \mu}{\partial x}\right) \delta x=\left(\sum_{i=1}^{n} \frac{\partial P(x)^{T}}{\partial x_{i}} \delta x_{i}\right) \mu=\left[\frac{\partial P(x)^{T}}{\partial x_{1}} \mu|\cdots| \frac{\partial P(x)^{T}}{\partial x_{n}} \mu\right] \delta x,
$$

which can be also considered as the Jacobian of the vector-valued function $P^{T}(x) \mu$ with respect to $x$. Equivalently, it is obtained by concatenating column vectors that are given by the matrixvector product of the matrix $\frac{\partial P(x)^{T}}{\partial x_{i}}$ and the vector $\mu$.

To impose this constraint on the infinitesimal variations explicitly, we decompose the variation $\delta \mu$ into the sum of two orthogonal components: one component in $\mathrm{T}_{x}^{*} \mathrm{M}$, namely $\delta \mu^{M}=$ $P(x)^{T} \delta \mu$, and the other component orthogonal to $\mathrm{T}_{x}^{*} \mathrm{M}$, namely $\delta \mu^{C}=\left(I_{n \times n}-P(x)^{T}\right) \delta \mu$. Then, satisfying the constrained variation implies

$$
\delta \mu^{C}=\left(I_{n \times n}-P(x)^{T}\right) \delta \mu=\left(\frac{\partial P^{T}(x) \mu}{\partial x}\right) \delta x .
$$

From Hamilton's phase space variational principle, $(x, \mu):\left[t_{0}, t_{f}\right] \rightarrow \mathrm{T}^{*} \mathrm{M}$ has the property that $\delta \mathfrak{G}=0$ for all continuous infinitesimal variations $\delta x:\left[t_{0}, t_{f}\right] \rightarrow \mathbb{R}^{n}, \delta \mu:\left[t_{0}, t_{f}\right] \rightarrow \mathbb{R}^{n}$, that satisfy $(\delta x(t), \delta \mu(t)) \in \mathrm{T}_{(x(t), \mu(t))} \mathbf{T}^{*} \mathrm{M}$ and $\delta x\left(t_{0}\right)=\delta x\left(t_{f}\right)=0$. This yields the following Hamilton's equations.

Proposition 2. Consider a differentiable manifold $\mathrm{M}$ embedded in $\mathbb{R}^{n}$. Hamilton's equations for a Hamiltonian $H: \mathrm{T}^{*} \mathrm{M} \rightarrow \mathbb{R}^{1}$ can be written as

$$
\dot{x}=P(x) \frac{\partial H(x, \mu)}{\partial \mu},
$$




\section{IMA Conference on Mathematics of Robotics}

9 - 11 September 2015, St Anne's College, University of Oxford

LAGRANGIAN AND HAMILTONIAN DYNAMICS ON AN EMBEDDED MANIFOLD

$$
\begin{aligned}
\dot{\mu}=- & P^{T}(x) \frac{\partial H(x, \mu)}{\partial x}+\left\{P^{T}(x)\left(\frac{\partial P^{T}(x) \mu}{\partial x}\right)^{T} P(x)\right. \\
& \left.+\left(\frac{\partial P^{T}(x) \mu}{\partial x}\right) P(x)-P^{T}(x)\left(\frac{\partial P^{T}(x) \mu}{\partial x}\right)^{T}\right\} \frac{\partial H(x, \mu)}{\partial \mu} .
\end{aligned}
$$

Furthermore, the Hamiltonian is preserved along any solution of Hamilton's equations, if it is time-invariant.

Proof. Decomposing $\delta \mu$ as $\delta \mu^{M}+\delta \mu^{C}$ and substituting (2.6), the infinitesimal variation of the action integral can be written as

$$
\begin{aligned}
\delta \mathfrak{G}=\int_{t_{0}}^{t_{f}}\left(-\dot{\mu}-\frac{\partial H(x, \mu)}{\partial x}+\left(\frac{\partial P^{T}(x) \mu}{\partial x}\right)^{T}\left(\dot{x}-\frac{\partial H(x, \mu)}{\partial \mu}\right)\right) \cdot \delta x \\
+P(x)\left(\dot{x}-\frac{\partial H(x, \mu)}{\partial \mu}\right) \cdot \delta \mu d t .
\end{aligned}
$$

Then, the fundamental lemma of the calculus of variations yields

$$
\begin{aligned}
& 0=P^{T}(x)\left(-\dot{\mu}-\frac{\partial H(x, \mu)}{\partial x}+\left(\frac{\partial P^{T}(x) \mu}{\partial x}\right)^{T}\left(\dot{x}-\frac{\partial H(x, \mu)}{\partial \mu}\right)\right), \\
& 0=P(x)\left(\dot{x}-\frac{\partial H(x, \mu)}{\partial \mu}\right) .
\end{aligned}
$$

Since $\dot{x} \in \mathrm{T}_{x} \mathrm{M}$ by definition, it follows that $P(x) \dot{x}=\dot{x}$. This yields (2.7) from the second equation above. The first equation (2.9) is incomplete since it only determines the component of $\dot{\mu}$ projected onto $\mathrm{T}_{x}^{*} \mathrm{M}$. The remaining component is determined by taking the time derivative of $\left(I_{n \times n}-P(x)^{T}\right) \mu=0$ to obtain

$$
-\left(\frac{\partial P^{T}(x) \mu}{\partial x}\right) \dot{x}+\left(I_{n \times n}-P^{T}(x)\right) \dot{\mu}=0
$$

which yields

$$
\left(I_{n \times n}-P^{T}(x)\right) \dot{\mu}=\left(\frac{\partial P^{T}(x) \mu}{\partial x}\right) \dot{x}=\left(\frac{\partial P^{T}(x) \mu}{\partial x}\right) P(x) \frac{\partial H(x, \mu)}{\partial \mu} .
$$

Combining (2.9) with (2.10), we obtain

$$
\begin{aligned}
\dot{\mu} & =P(x)^{T} \dot{\mu}+\left(I_{n \times n}-P(x)^{T}\right) \dot{\mu} \\
& =-P^{T}(x) \frac{\partial H(x, \mu)}{\partial x}+P^{T}(x)\left(\frac{\partial P^{T}(x) \mu}{\partial x}\right)^{T}\left(\dot{x}-\frac{\partial H(x, \mu)}{\partial \mu}\right)+\left(\frac{\partial P^{T}(x) \mu}{\partial x}\right) \dot{x} .
\end{aligned}
$$

Rearranging this with (2.7) yields (2.8).

Decomposing $\dot{\mu}$ into $\dot{\mu}=\left(I-P(x)^{T}\right) \dot{\mu}+P(x)^{T} \dot{\mu}$, the time-derivative of the Hamiltonian is written as

$$
\begin{aligned}
\frac{d H(x, \mu)}{d t}= & \frac{\partial H(x, \mu)}{\partial x} \cdot \dot{x}+P^{T}(x) \frac{\partial H(x, \mu)}{\partial \mu} \cdot P^{T}(x) \dot{\mu} \\
& +\left(I-P(x)^{T}\right) \frac{\partial H(x, \mu)}{\partial \mu} \cdot\left(I-P(x)^{T}\right) \dot{\mu}
\end{aligned}
$$

Substituting (2.9), (2.10), and (2.7), and rearranging using the symmetry of the projection, namely 


\section{IMA Conference on Mathematics of Robotics}

\section{9-11 September 2015, St Anne's College, University of Oxford}

Global Formulations of Lagrangian and Hamiltonian Dynamics on Embedded Manifolds

$P(x) P^{T}(x)=P^{T}(x)=P(x)$, we obtain $\frac{d H}{d t}=0$, which shows the conservation of the Hamiltonian.

Thus Hamilton's equations (2.7) and (2.8) describe the Hamiltonian flow in terms of the evolution of $(x, \mu) \in \mathrm{T}^{*} \mathrm{M}$.

\section{Lagrangian and Hamiltonian Dynamics for Specific Manifolds}

Lagrangian and Hamiltonian dynamics developed for an arbitrary embedded manifold in the prior section can be applied to a wide class of mechanical or robotic systems in a unified way. Here, we illustrate Lagrangian and Hamiltonian systems evolving on a subspace, the one-sphere, the two-sphere, and the special orthogonal group.

\subsection{Dynamics on a subspace $\mathrm{M}$ of $\mathbb{R}^{n}$}

Let $A \in \mathbb{R}^{m \times n}$ have rank $m$. The configuration manifold $\mathrm{M}=\left\{x \in \mathbb{R}^{n}: A x=0\right\}$ is a subspace of $\mathbb{R}^{n}$. The projection operator is the constant matrix $P(x)=I_{n \times n}-A^{T}\left(A^{T} A\right)^{-1} A$ for any $x \in \mathrm{M}$. Consequently, the Euler-Lagrange equation (2.3) reduces to

$$
\left[I_{n \times n}-A^{T}\left(A^{T} A\right)^{-1} A\right]\left\{\frac{d}{d t} \frac{\partial L}{\partial \dot{x}}-\frac{\partial L}{\partial x}\right\}=0 .
$$

The Legendre transformation yields $\mu=\left[I_{n \times n}-A^{T}\left(A^{T} A\right)^{-1} A\right] \frac{\partial L}{\partial \dot{x}}$. Hamilton's equations (2.7) and (2.8) simplify to

$$
\dot{x}=\left[I_{n \times n}-A^{T}\left(A^{T} A\right)^{-1} A\right] \frac{\partial H}{\partial \mu}, \quad \dot{\mu}=-\left[I_{n \times n}-A^{T}\left(A^{T} A\right)^{-1} A\right] \frac{\partial H}{\partial x} .
$$

\subsection{Dynamics on $\mathrm{M}=\mathrm{S}^{1}$}

Suppose the configuration manifold is given by the one-sphere, $M=S^{1}=\left\{x \in \mathbb{R}^{2} \mid\|x\|=1\right\}$. The projection operator at $x \in \mathrm{S}^{1}$ corresponds to the orthogonal projection onto the line normal to $x$, and it is given by $P(x)=I_{2 \times 2}-x x^{T}$. From (2.3), the Euler-Lagrange equation on $\mathrm{M}=\mathrm{S}^{1}$ is given by

$$
\left(I_{2 \times 2}-x x^{T}\right)\left\{\frac{d}{d t}\left(\frac{\partial L(x, \dot{x})}{\partial \dot{x}}\right)-\frac{\partial L(x, \dot{x})}{\partial x}\right\}=0 .
$$

The Legendre transformation yields $\mu=\left(I_{2 \times 2}-x x^{T}\right) \frac{\partial L}{\partial \dot{x}}$. The derivative of the projection operator defined by $(2.5)$ is

$$
\left(\frac{\partial P^{T}(x) \mu}{\partial x}\right) \delta x=\frac{\partial\left(I_{2 \times 2}-x x^{T}\right) \mu}{\partial x} \delta x=-\delta x x^{T} \mu-x \delta x^{T} \mu=-\left(x \mu^{T}\right) \delta x,
$$

where we have used the fact that $x^{T} \mu=0$. Substituting this into (2.8) and using the fact that $\mu^{T} x=0$ and $x^{T} x=1$, repeatedly, Hamillton's equations on $\mathrm{M}=\mathrm{S}^{1}$ are given by

$$
\dot{x}=\left(I_{2 \times 2}-x x^{T}\right) \frac{\partial H(x, \mu)}{\partial \mu}, \quad \dot{\mu}=-\left(I_{2 \times 2}-x x^{T}\right) \frac{\partial H(x, \mu)}{\partial x}+\left(\mu x^{T}-x \mu^{T}\right) \frac{\partial H(x, \mu)}{\partial \mu} .
$$

\subsection{Dynamics on $\mathrm{M}=\mathrm{S}^{2}$}

Suppose the configuration manifold is given by the two-sphere, $\mathrm{M}=\mathrm{S}^{2}=\left\{x \in \mathbb{R}^{3} \mid\|x\|=1\right\}$. The projection operator at $x \in \mathrm{S}^{2}$ is given by $P(x)=I_{3 \times 3}-x x^{T}$. Similar to the prior subsection, 


\section{IMA Conference on Mathematics of Robotics}

9 - 11 September 2015, St Anne's College, University of Oxford

LAGRANGIAN AND HAMILTONIAN DYNAMICS FOR SPECIFIC MANIFOLDS

the Euler-Lagrange equation on $\mathrm{M}=\mathrm{S}^{2}$ is

$$
\left(I_{3 \times 3}-x x^{T}\right)\left\{\frac{d}{d t}\left(\frac{\partial L(x, \dot{x})}{\partial \dot{x}}\right)-\frac{\partial L(x, \dot{x})}{\partial x}\right\}=0 .
$$

The Legendre transformation yields $\mu=\left(I_{3 \times 3}-x x^{T}\right) \frac{\partial L}{\partial \dot{x}}$. Hamilton's equations on $\mathrm{S}^{2}$ are written as

$$
\dot{x}=\left(I_{3 \times 3}-x x^{T}\right) \frac{\partial H(x, \mu)}{\partial \mu}, \quad \dot{\mu}=-\left(I_{3 \times 3}-x x^{T}\right) \frac{\partial H(x, \mu)}{\partial x}+(x \times \mu) \times \frac{\partial H(x, \mu)}{\partial \mu},
$$

where we have used the identity $\left(\mu x^{T}-x \mu^{T}\right) z=(x \times \mu) \times z$ for any $x, z, \mu \in \mathbb{R}^{3}$.

\subsection{Dynamics on $\mathrm{M}=\mathrm{SO}(3)$}

Suppose that the configuration manifold is the special orthogonal group, $\mathrm{SO}(3)=\left\{R \in \mathbb{R}^{3 \times 3} \mid R^{T} R=\right.$ $I_{3 \times 3}$, $\left.\operatorname{det}[R]=1\right\}$. The tangent space at $R$ is given by $\mathrm{T}_{R} \mathrm{SO}(3)=\left\{V \in \mathbb{R}^{3 \times 3} \mid R^{T} V+V^{T} R=\right.$ $0\}$. For any $V \in \mathrm{T}_{R} \mathrm{SO}(3)$, there exists $v \in \mathbb{R}^{3}$ such that $V=R \hat{v}$, where the hat map $\wedge$ transforms a $3 \times 1$ vector into a $3 \times 3$ skew-symmetric matrix such that $\hat{x} y=x \times y$ for any $x, y \in \mathbb{R}^{3}$ (Marsden 1999). The inverse of the hat map is denoted by the vee map, $\mathrm{V}$.

Instead of considering that $\mathrm{SO}(3)$ is embedded in $\mathbb{R}^{9}$, we assume that the embedding space of $\mathrm{SO}(3)$ is $\mathbb{R}^{3 \times 3}$. The definitions of the inner product and the projection operator are first generalized to $\mathbb{R}^{3 \times 3}$ as follows. The inner-product is defined as an element-wise operation. More explicitly, for $V, W \in \mathrm{T}_{R} \mathrm{SO}(3)$,

$$
V \cdot W=\operatorname{tr}\left[V^{T} W\right]=\sum_{i, j=1}^{3} V_{i j} W_{i j}
$$

The projection operator acted on $Y \in \mathbb{R}^{3 \times 3}$ can be written as

$$
P(R, Y)=\frac{1}{2} R\left(R^{T} Y-Y^{T} R\right)=\frac{1}{2}\left(Y-R Y^{T} R\right),
$$

which is clearly in $\mathrm{T}_{R} \mathrm{SO}(3)$. The projection is orthogonal since

$$
(Y-P(R, Y)) \cdot R \hat{z}=\frac{1}{2} \operatorname{tr}\left[\left(Y+R Y^{T} R\right) \hat{z} R^{T}\right]=\frac{1}{2} \operatorname{tr}\left[\left(R^{T} Y+Y^{T} R\right) \hat{z}\right]=0,
$$

for any $z \in \mathbb{R}^{3}$, where we have used the facts that the trace is invariant under transpose and cyclic permutation, and the trace of the product of any symmetric matrix and any compatible skew-symmetric matrix is zero. It is also symmetric since for any $Z \in \mathbb{R}^{3 \times 3}$,

$$
Z \cdot P(R, Y)=\frac{1}{2} \operatorname{tr}\left[\left(Y-R Y^{T} R\right) Z^{T}\right]=\frac{1}{2} \operatorname{tr}\left[Y^{T}\left(Z-R Z^{T} R\right)\right]=Y \cdot P(R, Z) .
$$

To describe the proposed approach more clearly and explicitly, the attitude dynamics of a free rigid body is considered. Let $J \in \mathbb{R}^{3 \times 3}$ be the moment of inertia matrix of the rigid body, and define a non-standard inertia matrix $J_{d}=\frac{1}{2} \operatorname{tr}[J] I_{3 \times 3}-J$. The Lagrangian of the rigid body corresponding to the rotational kinetic energy can be written as

$$
L(R, \dot{R})=\frac{1}{2} \operatorname{tr}\left[\dot{R} J_{d} \dot{R}^{T}\right]=\frac{1}{2} \dot{R} \cdot \dot{R} J_{d}
$$

which is identical to the common expression $\frac{1}{2} \Omega^{T} J \Omega$ where $\Omega=\left(R^{T} \dot{R}\right)^{\vee} \in \mathbb{R}^{3}$ denotes the angular velocity of the rigid body (Lee 2007).

We have $\frac{\partial L}{\partial \dot{R}}=\dot{R} J_{d}$, where $\frac{\partial L}{\partial \dot{R}} \in \mathbb{R}^{3 \times 3}$ are defined such that its $(i, j)$-th element corresponds 


\section{IMA Conference on Mathematics of Robotics}

\section{9-11 September 2015, St Anne's College, University of Oxford}

Global Formulations of Lagrangian and Hamiltonian Dynamics on Embedded Manifolds

to the derivative of $L$ with respect to the $(i, j)$-th element of $R$. From (2.3) and (3.7), the EulerLagrange equation is given by

$$
\ddot{R} J_{d}-R J_{d} \ddot{R}^{T} R=0 .
$$

Next, we show that this is equivalent to the common Euler's equation for a rigid body, written in terms of the standard inertia matrix $J$ and the angular velocity $\Omega$. Since $\dot{R}=R \hat{\Omega}$, we have $\ddot{R}=R \hat{\Omega}^{2}+R \hat{\dot{\Omega}}$. Therefore, $R^{T} \ddot{R}=\hat{\Omega}^{2}+\hat{\dot{\Omega}}$. Left-multiplying both sides of the above expression with $R^{T}$, and substituting this,

$$
\hat{\dot{\Omega}} J_{d}+J_{d} \hat{\dot{\Omega}}+\hat{\Omega}^{2} J_{d}-J_{d} \hat{\Omega}^{2}=0 .
$$

Using the identities of the hat map, we have $\hat{\dot{\Omega}} J_{d}+J_{d} \hat{\dot{\Omega}}=\left\{\left(\operatorname{tr}\left[J_{d}\right] I_{3 \times 3}-J_{d}\right) \dot{\Omega}\right\}^{\wedge}=(J \dot{\Omega})^{\wedge}$, and $\hat{\Omega}^{2} J_{d}-J_{d} \hat{\Omega}^{2}=\widehat{J_{d} \Omega} \hat{\Omega}-\hat{\Omega} \widehat{J_{d} \Omega}=-\widehat{J \Omega} \hat{\Omega}+\hat{\Omega} \widehat{J \Omega}=(\Omega \times J \Omega)^{\wedge}$. Therefore, the above equation reduces to Euler's equation,

$$
J \dot{\Omega}+\Omega \times J \Omega=0 .
$$

These results can be also generalized to a complicated manifold that is composed of the products of an arbitrary number of the one-spheres, the two-spheres, and the special orthogonal groups.

\section{Acknowledgements}

TL has been supported in part by NSF under grants CMMI-1243000, CMMI-1335008, and CNS1337722. ML has been supported in part by NSF under grants DMS-1010687, CMMI-1029445, DMS-1065972, CMMI-1334759, DMS-1411792, DMS-1345013.

\section{REFERENCES}

V. ARnold, Mathematical Methods of Classical Mechanics, Springer, 1989.

A. BLOCH, Nonholonomic Mechanics and Control, vol. 24 of Interdisciplinary Applied Mathematics, Springer-Verlag, 2003.

F. BlOCH, Nuclear induction, Physical Review, 70 (1946).

F. Bullo AND A. LEWIS, Geometric control of mechanical systems, vol. 49 of Texts in Applied Mathematics, Springer-Verlag, New York, 2005. Modeling, analysis, and design for simple mechanical control systems.

D. Holm, T. Schmah, And C. Stoica, Geometric Mechanics and Symmetry, Oxford University Press, 2009.

T. LeE, M. LEOK, AND N. MCClamRoch, Dynamics and control of a chain pendulum on a cart, in Proceedings of the IEEE Conference on Decision and Control, Maui, HI, Dec. 2012, pp. 2502-2508.

T. LEE, M. LEOK, AND N. H. MCCLAMROCH, Lie group variational integrators for the full body problem, Computer Methods in Applied Mechanics and Engineering, 196 (2007), pp. 2907-2924.

T. LEE, M. LEOK, AND N. H. MCCLAMROCH, Lagrangian mechanics and variational integrators on two-spheres, International Journal for Numerical Methods in Engineering, 79 (2009), pp. 1147-1174.

J. Marsden, Lectures on Mechanics, London Mathematical Society Lecture Note Series 174, Cambridge University Press, 1992.

J. Marsden, R. Montgomery, And T. Ratiu, Reduction, Symmetry and Phases in Mechanics, American Mathematical Society, 1990.

J. MARSDEN AND T. RATIU, Introduction to Mechanics and Symmetry, vol. 17 of Texts in Applied Mathematics, Springer-Verlag, second ed., 1999.

R. Murray, Z. Li, AND S. SASTRY, A Mathematical Introduction to Robotic Manipulation, CRC Press, 1993. 